\title{
LIOUVILLIAN FIRST INTEGRALS OF DIFFERENTIAL EQUATIONS
}

\author{
MICHAEL F. SINGER
}

\begin{abstract}
Liouvillian functions are functions that are built up from rational functions using exponentiation, integration, and algebraic functions. We show that if a system of differential equations has a generic solution that satisfies a liouvillian relation, that is, there is a liouvillian function of several variables vanishing on the curve defined by this solution, then the system has a liouvillian first integral, that is a nonconstant liouvillian function that is constant on solution curves in some nonempty open set. We can refine this result in special cases to show that the first integral must be of a very special form. For example, we can show that if the system $d x / d z=P(x, y), d y / d z=Q(x, y)$ has a solution $(x(z), y(z))$ satisfying a liouvillian relation then either $x(z)$ and $y(z)$ are algebraically dependent or the system has a liouvillian first integral of the form $F(x, y)=\int R Q d x-R P d y$ where $R=\exp \left(\int U d x+V d y\right)$ and $U$ and $V$ rational functions of $x$ and $y$. We can also reprove an old result of Ritt stating that a second order linear differential equation has a nonconstant solution satisfying a liouvillian relation if and only if all of its solutions are liouvillian.
\end{abstract}

\section{INTRODUCTION}

In elementary courses on differential equations we consider systems of the form

$$
\begin{aligned}
& \frac{d x}{d z}=P(x, y), \\
& \frac{d y}{d z}=Q(x, y),
\end{aligned}
$$

where $P$ and $Q$ are polynomials in $\mathbb{C}(x, y), \mathbb{C}$ being the complex numbers. We learn that although we cannot always explicitly solve this system, we are occasionally able to find first integrals, that is nonconstant functions $F(x, y)$, analytic on some nonempty open set in $\mathbb{C}^{2}$, that are constant on solution curves in this set. To do this we consider the differential form $Q(x, y) d x-P(x, y) d y$. If $\partial P / \partial x=-\partial Q / \partial y$, then $F(x, y)=\int Q d x-P d y$ will be a first integral. If $\partial P / \partial x \neq-\partial Q / \partial y$, we are taught ad hoc methods to find an integrating factor, that is a function $R(x, y)$ such that $\partial(R P) / \partial x=-\partial(R Q) / \partial y$. In case we can find such a function $R, F(x, y)=\int R Q d x-R P d y$ will be a

Received by the editors October 24, 1988 and, in revised form, July 26, 1990.

1991 Mathematics Subject Classification. Primary 12H05.

The preparation of this paper was partially supported by NSF Grants DMS 84-20075 and DMS 88-03109. 
first integral. For example, if $(\partial Q / \partial x+\partial P / \partial y) / P$ is independent of $y$, then $R=\exp \left(\int(\partial Q / \partial x+\partial P / \partial y) / P d x\right.$ will be an integrating factor. A natural question arises: When does the system (1) have a first integral that can be expressed in terms of the functions known to a calculus student (that is those functions that can be built up using integration, exponentiation, and algebraic operations from the rational functions; see $\$ 2$ for a precise definition of these functions-the liouvillian functions) and how does one find such an integral? To answer the first part of this question, we show in this paper the following

Theorem 1. Let $P(x, y)$ and $Q(x, y)$ be polynomials in two variables and let $(\bar{x}(t), \bar{y}(t))$ be a solution of (1) which is analytic on some nonempty open set $\mathscr{O} \subset \mathbb{C}$. If there exists a nonzero liouvillian function $F(x, y)$ analytic on some open set in $\mathbb{C}^{2}$ containing $\mathscr{G}=\{(\bar{x}(z), \bar{y}(z)) \mid z \in \mathscr{O}\}$ and vanishing on $\mathscr{G}$, then either there is a nonzero polynomial $G$ such that $G(x(z), y(z))=0$ or the differential form $Q(x, y) d x-P(x, y) d y$ has an integrating factor of the form

$$
R(x, y)=\exp \left[\int_{\left(x_{0}, y_{0}\right)}^{(x, y)} U(x, y) d x+V(x, y) d y\right],
$$

where $U$ and $V$ are rational functions with $\partial U / \partial y=\partial V / \partial x$ so that this latter line integral is well defined.

We will deduce the following corollary.

Corollary. The system of differential equations (1) has a liouvillian first integral if and only if the differential form $Q(x, y) d x-P(x, y) d y$ has an integrating factor of the form (2), in which case

$$
F(x, y)=\int_{\left(x_{0}, y_{0}\right)}^{(x, y)} R(x, y) Q(x, y) d x-R(x, y) P(x, y) d y
$$

is a liouvillian first integral.

Note that (1) may have a liouvillian first integral without having nonconstant liouvillian solutions. An example of this is the system $d x / d z=x y-$ $x^{2}, d y / d z=y^{2}$ which has the liouvillian first integral $y-\exp (y / x)$ [ROS69]. In contrast to this we reprove the following result of Ritt [RITT27] or [RITT48].

Theorem 2. Let $\bar{y}(z)$ be a nonzero solution of $y^{\prime \prime}+p(z) y^{\prime}+q(z) y=0$, where $p(z)$ and $q(z)$ are liouvillian functions of $z$, and assume that $\bar{y}(z), p(z)$, and $q(z)$ are analytic on some open set $\mathscr{O}$. If there is a nonzero liouvillian function $F$ of three variables analytic on some open set $\mathscr{U}$ in $\mathbb{C}^{3}$ containing $\mathscr{G}=\{(z, p(z), q(z)) \mid z \in \mathscr{O}\}$ and vanishing on $\mathscr{G}$, then $y(z)$ is a liouvillian function of $z$.

As a corollary we have the following result concerning liouvillian first integrals. By a liouvillian first integral of an $n$th order differential equation, we mean a nonzero liouvillian function $F$ of $n$ variables analytic in a nonempty open subset $\mathscr{U}$ of $\mathbb{C}^{n}$ such that $F\left(z, \bar{y}(z), \bar{y}^{\prime}(z), \ldots, \bar{y}^{(n-1)}(z)\right)$ is constant for any solution $\bar{y}(z)$ of the differential equation whenever $\left(z, \bar{y}(z), \bar{y}^{\prime}(z), \ldots\right.$, $\left.\bar{y}^{(n-1)}(z)\right)$ is in $\mathscr{U}$.

Corollary. Let $p(z)$ and $q(z)$ be liouvillian functions. If $L(y)=y^{\prime \prime}+p(z) y^{\prime}+$ $q(z) y=0$ has a liouvillian first integral then all solutions of $L(y)=0$ are 
liouvillian. If $L(y)=0$ has a nonzero liouvillian solution, then this equation has a liouvillian first integral.

The rest of this paper is organized as follows. In $\S 2$, we give a formal definition of a liouvillian function, liouvillian relation, and liouvillian first integral. We show that if a system of differential equations has a sufficiently generic solution that satisfies a liouvillian relation, then the system has a liouvillian first integral (Proposition 2.1). We also derive an algebraic consequence (Proposition 2.2) of such an event. Proposition 2.2 will be the main technical tool of this paper. Section 3 is devoted to liouvillian first integrals of planar vector fields, a proof of Theorem 1 and its corollary, and a discussion of algorithmic considerations. Section 4 is devoted to liouvillian first integrals of linear differential equations and a proof of Theorem 2 and its corollary. In the appendix we give a proof of a theorem of Darboux that is used in this paper. I would like to thank the referee for extensive comments, corrections, and suggestions regarding both the content and style of earlier versions of this paper.

\section{LIOUVILLIAN RELATIONS AND FIRST INTEGRALS}

We begin this section with the formal definition of a liouvillian function. This is given in the setting of differential algebra (cf. [KOL73 or KAP57]). Let $(k, \Delta)$ denote a differential field of characteristic zero with a given set of commuting derivations $\Delta=\left\{\delta_{i}\right\}$ (all differential fields in this paper will be assumed to have commuting sets of derivations). A differential field $(M, \Delta)$ is a liouvillian extension of $(K, \Delta)$ if there is a tower of fields $k=K_{0} \subset$ $K_{1} \subset \cdots \subset K_{m}=M$ where each $K_{i}=K_{i-1}\left(t_{i}\right)$ with either (i) $\delta t_{i} \in K_{i-1}$ for all $\delta \in \Delta$, or (ii) $\delta t_{i} / t_{i} \in K_{i-1}$ for all $\delta \in \Delta$, or (iii) $t_{i}$ is algebraic over $K_{i-1}$. We say that $k=K_{0} \subset \cdots \subset K_{m}=M$ is the defining tower of $M$ and define the length of the defining tower to be the integer $m$. The constants of $(k, \Delta)$, that is, all those elements annihilated by all $\delta$ in $\Delta$, will be denoted by $C(k, \Delta)$. Let $k=\mathbb{C}\left(y_{1}, \ldots, y_{n}\right)$, where $y_{1}, \ldots, y_{n}$ are indeterminates and let $\Delta=\left\{\partial / \partial y_{1}, \ldots, \partial / \partial y_{n}\right\}$. Let $(M, \Delta)$ be a liouvillian extension of $(k, \Delta)$ such that the field of constants of $(M, \Delta)=\mathbb{C}$. One can see by induction that each element of $M$ represents a function analytic on a dense open set on $\mathbb{C}^{n}$ (in fact, any differential field finitely generated over $\mathbb{Q}$ is isomorphic to a field of functions meromorphic in some domain [SEI58, SEI69], but what we need is easier to prove). We call such a function a liouvillian function of $n$ variables. Note that it can happen that an element of $M$ can represent a function that is analytic on a larger set than the $t_{i}$ 's. For example, $\sin \left(\sqrt{y}_{1}\right)$ is analytic on all of $\mathbb{C}$ and lies in $\mathbb{C}\left(y_{1}, \sqrt{y}_{1}, \exp \left(\sqrt{y}_{1}\right)\right)$. We remark that if $\mathbb{C}\left(y_{1}, \ldots, y_{n}\right)=$ $K_{0} \subset \cdots \subset K_{m_{0}}=M_{0}$ and $\mathbb{C}\left(y_{1}, \ldots, y_{n}\right)=K_{0}^{\prime} \subset \cdots \subset K_{m_{1}}^{\prime}=M_{1}$ are liouvillian extensions of $\mathbb{C}\left(y_{1}, \ldots, y_{n}\right)$ with $C\left(M_{0}, \Delta\right)=C\left(M_{1}, \Delta\right)=\mathbb{C}$, then the compositum $M=M_{0} M_{1}$ is also a liouvillian extension of $\mathbb{C}\left(y_{1}, \ldots, y_{n}\right)$. To see this just consider the tower $K_{0} \subset \cdots \subset K_{m_{0}} \subset K_{m_{0}} K_{1}^{\prime} \subset \cdots \subset K_{m_{0}} K_{m_{1}}^{\prime}=$ $M_{0} M_{1}=M$. Furthermore, $C(M, \Delta)=\mathbb{C}$. To see this, note that this field is finitely generated over $\mathbb{C}$ by elements each of which is analytic on a dense open set. Therefore, there is a nonempty open set in which all the elements of $M$ are meromorphic. Therefore the only constants are in $\mathbb{C}$. This implies that given a finite number of liouvillian functions of $n$ variables, there exists a liouvillian extension $M$ of $\mathbb{C}\left(y_{1}, \ldots, y_{n}\right)$ containing all of them and satisfying $C(M, \Delta)=\mathbb{C}$. 
In what follows we will consider systems of differential equations of the form

$$
\begin{gathered}
\frac{d y_{1}}{d z}=f_{1}\left(y_{1}, \ldots, y_{n}\right) \\
\ldots \\
\ldots \\
\frac{d y_{n}}{d z}=f_{n}\left(y_{1}, \ldots, y_{n}\right)
\end{gathered}
$$

where the $f_{i}$ are liouvillian functions analytic on some domain (= a connected, nonempty, open set) $\mathscr{U}_{0} \subset \mathbb{C}^{n}$. Let $(k, \Delta)$ be the differential extension of $\mathbb{C}\left(y_{1}, \ldots, y_{n}\right)$ generated by the $f_{i}$ and their partial derivatives of all orders with $\Delta=\left\{\partial / \partial y_{i}\right\}$. Note that every element of $k$ can be written as the quotient of elements in $k$ that are analytic in $\mathscr{U}_{0}$. In other words, $k$ is the quotient field of $\mathscr{H}\left(\mathscr{U}_{0}\right) \cap k$, where $\mathscr{H}\left(\mathscr{U}_{0}\right)$ is the ring of analytic functions on $\mathscr{U}_{0}$. Let $\bar{y}_{1}(z), \ldots, \bar{y}_{n}(z)$ be a solution of (3) analytic in some domain $\mathscr{O}_{0} \subset \mathbb{C}$ and assume $\mathscr{G}_{0}=\left\{\left(\bar{y}_{1}(z), \ldots, \bar{y}_{n}(z)\right) \mid z \in \mathscr{O}_{0}\right\} \subset \mathscr{U}_{0}$. We shall also make the following assumption regarding this solution: each element $G$ in $k$ is analytic and not identically zero on a nonempty open dense subset $\mathscr{O}_{G}$ of $\mathscr{G}_{0}$. We shall refer to this as the basic assumption for $\bar{y}_{1}(z), \ldots, \bar{y}_{n}(z)$. This basic assumption is easily verified in two situations which we now describe. First, let us assume that the $f_{i}$ appearing in (3) are polynomials in the $y_{j}$ with coefficients in $\mathbb{C}$; this will be the situation in Theorem 1 . Let $\bar{y}_{1}(z), \ldots, \bar{y}_{n}(z)$ be a solution analytic in some nonempty open set $\mathscr{O}_{0} \subset \mathbb{C}$. In this case we let $k=\mathbb{C}\left(y_{1}, \ldots, y_{n}\right)$. If $\bar{y}_{1}(z), \ldots, \bar{y}_{n}(z)$ are algebraically independent over $\mathbb{C}$, then this solution satisfies the basic assumption. To see this, note that no nonzero polynomial with complex coefficients can vanish identically on $\mathscr{G}_{0}$. Therefore each rational function in $k$ is analytic and nonzero on an open dense subset of $\mathscr{G}_{0}$. Second, let $f_{n}=1$ (so $\bar{y}_{n}$ can be assumed to be $z$ ) and let $f_{1}, \ldots, f_{n-1}$ be polynomials whose coefficients are liouvillian functions of one variable $y_{n}$; this will be the situation in Theorem 2 . Let $\left(k_{0},\left\{\partial / \partial y_{n}\right\}\right)$ be the differential field generated by these coefficients and let $k=k_{0}\left(y_{1}, \ldots, y_{n-1}\right)$. If $\bar{y}_{1}(z), \ldots, \bar{y}_{n-1}(z)$ are algebraically independent over $k_{0}$ and form a solution of (3), then one can see as above that this solution satisfies the basic assumption.

We say that $\bar{y}_{1}(z), \ldots, \bar{y}_{n}(z)$ satisfy a liouvillian relation if there is a liouvillian function $F\left(y_{1}, \ldots, y_{n}\right)$, analytic on some open subset of $\mathbb{C}^{n}$ containing $\mathscr{G}$, such that $\left.F\right|_{\mathscr{G}} \equiv 0$. Notice that we do not insist that the elements, generating a defining liouvillian tower containing $F$, be defined in a neighborhood of $\mathscr{G}$, but only that $F$ be. By the above remarks, we may assume that $F$ belongs to a liouvillian extension $M$ of $k$ with $C(M, \Delta)=\mathbb{C}$. Clearly, if $\bar{y}_{1}(z), \ldots, \bar{y}_{n}(z)$ are algebraically dependent over $\mathbb{C}$, then they satisfy a liouvillian relation. We say that a nonconstant function $G\left(y_{1}, \ldots, y_{n}\right)$, analytic on some nonempty open subset $\mathscr{U}$ of $\mathbb{C}^{n}$ is a first integral of (3) if, for any solution $\overline{\bar{y}}_{1}(z), \ldots, \overline{\bar{y}}_{n}(z)$ defined on an open set $\mathscr{O}_{0} \subset \mathbb{C}^{n}$ with $\mathscr{G}_{0}=\left\{\left(\overline{\bar{y}}_{1}(z), \ldots, \overline{\bar{y}}_{n}(z)\right) \mid z \in \mathscr{O}_{0}\right\} \subset \mathscr{U}$, there exists a complex number $c$ such that $\left.G\right|_{\mathscr{G}_{0}} \equiv c$. This latter condition is equivalent to the fact that $D G \equiv 0$ on $\mathscr{U}$ where $D=\sum f_{i}\left(y_{1}, \ldots, y_{n}\right) \partial / \partial y_{i}$. Note that $D$ defines a derivation on $k$ as well as on any differential extension of this field. The next proposition 
shows that for a solution of (3) satisfying the basic assumption, the existence of a liouvillian relation implies the existence of a liouvillian first integral for (3).

Proposition 2.1. Let $\bar{y}_{1}(z), \ldots, \bar{y}_{n}(z)$ be a solution of (3) analytic on some domain $\mathscr{O}_{0} \subset \mathbb{C}$ and let $\mathscr{G}_{0}=\left\{\left(\bar{y}_{1}(z), \ldots, \bar{y}_{n}(z)\right) \mid z \in \mathscr{O}_{0}\right\}$. Assume that $\bar{y}_{1}(z), \ldots, \bar{y}_{n}(z)$ satisfy the basic assumption and satisfy a liouvillian relation. Then there exists a liouvillian extension $M$ of $\left(k,\left\{\partial / \partial y_{1}, \ldots, \partial / \partial y_{n}\right\}\right)$ such that:

(i) There exists an open set $\mathscr{O} \subset \mathscr{O}_{0}$ and an open set $\mathscr{U} \subset \mathscr{U}_{0} \subset \mathbb{C}^{n}$ containing $\mathscr{G}=\left\{\left(\bar{y}_{1}(z), \ldots, \bar{y}_{n}(z)\right) \mid z \in \mathcal{O}\right\}$ such that the elements of $M$ are meromorphic in $\mathscr{U}$.

(ii) If $k=K_{0} \subset \cdots \subset K_{m}=M$ is the defining tower of $M$, then any element of $K_{m-1}$ is analytic and not identically zero on an open dense subset of $\mathscr{G}$. Therefore, the map sending $G\left(y_{1}, \ldots, y_{n}\right) \in K_{m-1}$ to $G\left(\bar{y}_{1}(z), \ldots, \bar{y}_{n}(z)\right)$ defines an isomorphism $\varphi$ of $K_{m-1}$ onto a differential field of functions of one variable meromorphic in $\mathscr{O}$ such that $d(\varphi(G)) / d z=\sum \varphi\left(\partial G / \partial y_{i}\right) \varphi\left(f_{i}\right)$.

(iii) There is a $W \in M$ such that $D W=0$ while $\partial W / \partial y_{j} \neq 0$ for some $j$. Proof. Let $\mathscr{L}$ be the set of triples $\left(\mathscr{O}^{\prime}, \mathscr{U}^{\prime}, F^{\prime}\right)$ where $\mathscr{O}^{\prime}$ is a nonempty subdomain of $\mathscr{O}$ such that $\mathscr{G}^{\prime}=\left\{\left(\bar{y}_{1}(z), \ldots, \bar{y}_{n}(z)\right) \mid z \in \mathscr{O}^{\prime}\right\} \subset \mathscr{U}^{\prime} \subset \mathbb{C}^{n}, \mathscr{U}^{\prime}$ a subdomain of $\mathscr{U}, F^{\prime}$ is a liouvillian function, analytic and not identically zero on $\mathscr{U}^{\prime}$ and $\left.F^{\prime}\right|_{\mathscr{G}} \equiv 0$. Since $\bar{y}_{1}(z), \ldots, \bar{y}_{n}(z)$ satisfy a liouvillian relation, $\mathscr{L}$ is not empty. Select an element $\left(\mathscr{O}^{\prime \prime}, \mathscr{U}^{\prime \prime}, F^{\prime \prime}\right)$ in $\mathscr{L}$ such that $F^{\prime \prime}$ belongs to a tower of minimal length and denote this tower by $k=K_{0} \subset \cdots \subset K_{m}$. Let $M=K_{m}$. We are now considering the functions in $k$ as functions on $\mathscr{U}^{\prime \prime}$ and the $y_{i}$ as functions on $\mathscr{O}^{\prime \prime}$. We will first verify that these restricted functions still satisfy the basic assumption. Let $G \in k$ and write $G=G_{1} / G_{2}$, where $G_{1}$ and $G_{2}$ are in $k$ and analytic in $\mathscr{U}_{0}$. If $G_{2}$ vanishes on a nonempty open subset of $\mathscr{G}^{\prime \prime}=\left\{\left(\bar{y}_{1}(z), \ldots, \bar{y}_{n}(z)\right) \mid z \in \mathscr{O}^{\prime}\right\} \subset \mathscr{G}_{0}$, then by the identity theorem, $G_{2}$ vanishes on all of $\mathscr{G}_{0}$. This contradicts the fact that the basic assumption holds for $\mathscr{O}_{0}$ and $\mathscr{U}_{0}$. Therefore, $G$ is analytic on a dense open subset of $\mathscr{G}^{\prime \prime}$. In a similar manner, one can show that $G$ is not identically zero on $\mathscr{G}^{\prime \prime}$ and so the restricted functions satisfy the basic assumption. We therefore may assume that $\mathscr{O}^{\prime \prime}=\mathscr{O}_{0}$ and $\mathscr{U}^{\prime \prime}=\mathscr{U}_{0}$. Note that this implies that $n>0$. We shall now show that (i) and (ii) hold for $K_{i}$ by induction on $i$. Assume that there exists a domain $\mathscr{O}_{i-1} \subset \mathscr{O}_{0}$ and a domain $\mathscr{U}_{i-1} \subset \mathscr{U}_{0}$ containing $\mathscr{G}_{i-1}=\left\{\left(\bar{y}_{1}(z), \ldots, \bar{y}_{n}(z)\right) \mid z \in \mathscr{O}_{i-1}\right\}$ such that any element of $K_{i-1}$ is meromorphic on $\mathscr{U}_{i-1}$ and analytic and not identically zero on an open dense subset of $\mathscr{G}_{i-1}$. Furthermore, assume that $K_{i-1}$ is the quotient field of $\mathscr{H}\left(\mathscr{U}_{i-1}\right) \cap K_{i-1}$. We wish to show that there are domains $\mathscr{O}_{i} \subset \mathscr{O}_{i-1}$ and $\mathscr{U}_{i} \subset \mathscr{U}_{i-1}$ satisfying similar properties with respect to $K_{i}$ (except if $i=m$, in which case we will not guarantee that elements are nonzero or analytic on a dense open subset of $\mathscr{G}_{m}=\mathscr{G}^{\prime}$ ).

First assume that $K_{i}=K_{i-1}(t)$ where $t$ is transcendental over $K_{i-1}$ and $\partial t / \partial y_{j} \in K_{i-1}$ for $j=1, \ldots, n$. For each $j$, there exists an open subset $\mathscr{U}^{j}$ of $\mathscr{U}_{i-1}$ such that $\partial t / \partial y_{j}$ is analytic in $\mathscr{U}^{j}$ and $\mathscr{U}^{j} \cap \mathscr{G}_{i-1}$ is dense in $\mathscr{G}_{i-1}$. Furthermore, some $\partial t / \partial y_{j}$ is nonzero in $\mathscr{U}^{j} \cap \mathscr{G}_{i-1} . \mathscr{U}=\bigcap_{j=1}^{n} \mathscr{U}^{j}$ is a nonzero open set intersecting $\mathscr{G}_{i-1} . t$ is analytic on a dense open subset of $\mathbb{C}^{n}$, so let $p \in \mathscr{U}$ be a point where $t$ is analytic such that for some $\varepsilon>0$, the $\varepsilon$-ball $B$ centered at $P$ is contained in $\mathscr{U}$ and $B \cap \mathscr{G}_{i-1}$ is not empty. 
Since each $\partial t / \partial y_{j}$ is analytic in $B$ and $\partial^{2} t / \partial y_{j} \partial y_{k}=\partial^{2} t / \partial y_{k} \partial y_{j}, t$ can be defined to be analytic in all of $B$ [KRA82, Lemma 6.3.4, p. 234]. Let the domain $\mathscr{O}_{i} \subset \mathscr{O}_{i-1}$ be chosen such that $\mathscr{G}_{i}=\left\{\left(\bar{y}_{1}(z), \ldots, \bar{y}_{n}(z)\right) \mid z \in \mathscr{O}_{i}\right\}$ is contained in $B$. We let $\mathscr{U}_{i}=B$. $t$ cannot be identically zero on $\mathscr{G}_{i}$ by the minimality of $N$. We now consider the functions of $K_{i-1}$ as being restricted to $\mathscr{U}_{i}$. As above, one can show that each $G$ in $K_{i-1}$ is analytic and not identically zero on a dense open subset of $\mathscr{G}_{i}$. Let $p(t)=a_{r} t^{r}+\cdots+a_{0} \in K_{i-1}[t] . p(t)$ will be meromorphic on $\mathscr{U}_{i}$ and analytic on a dense open subset of $\mathscr{G}_{i}$. If $i<m$ and $p(t)$ were zero on a nonempty open subset of $\mathscr{G}_{i}$, then we would contradict the minimality of $m$. If $G=p(t) / q(t)$ with $p(t), q(t) \in K_{i-1}[t]$, a similar argument shows that $G$ is meromorphic in $\mathscr{U}_{i}$ and analytic on a dense open subset of $\mathscr{G}_{i}$ and nonzero there if $i<m$. Finally, since $t$ is analytic in $\mathscr{U}_{i}, K_{i}$ is the quotient field of $\mathscr{H}\left(\mathscr{U}_{i}\right) \cap K_{i}$.

If $K_{i}=K_{i-1}(t)$ with $\partial t / \partial y_{j} / t \in K_{i-1}$ for each $j$ and $t$ is transcendental over $K_{i-1}$, we proceed as follows. Let $s_{j}=\partial t / \partial y_{j}$. Note that $\partial s_{j} / \partial y_{i}=$ $\partial s_{i} / \partial y_{j}$ for all $1 \leq i, j \leq n$, so we can find a function $v$ such that $\partial v / \partial y_{j}=s_{j}$ as above and let $t=\exp (v)$. Arguing as above gives us the conclusion in this case as well.

Finally assume that $K_{i}=K_{i-1}(t)$ with $t$ algebraic over $K_{i-1}$. Let $P(t)=$ $t^{r}+a_{r-1} t^{r-1}+\cdots+a_{0}$ be the minimum polynomial of $t$ over $K_{i-1}$. Using the induction hypothesis, we can assume that there is an open set $\mathscr{U}_{P}$ such that the $a_{j}$ are analytic in $\mathscr{U}_{P}$ and $\mathscr{U}_{P} \cap \mathscr{G}_{i-1}$ is dense in $\mathscr{G}_{i-1}$. Let $D$ be the discriminant of $P$ [LANG65, Chapter $5, \S 10] . D$ is a polynomial in the $a_{j}$ with constant coefficients so it will be analytic in $\mathscr{U}_{P}$. Since $P(t)$ is irreducible, $D$ does not vanish identically on $\mathscr{U}_{P}$ [LANG65, Chapter 5, $\S 10$, Proposition 4] and since $D \in K_{i-1}$ it does not vanish identically on $\mathscr{G}_{i-1}$. Therefore there exists a domain $\mathscr{O}_{i} \subset \mathscr{O}_{i-1}$ such that $\mathscr{G}_{i}=\left\{\left(\bar{y}_{1}(z), \ldots, \bar{y}_{n}(z) \mid z \in \mathscr{O}_{i}\right\} \subset \mathscr{U}_{i}\right.$, where $\mathscr{U}_{i}$ is a component of $\left\{\left(y_{1}, \ldots, y_{n}\right) \in \mathscr{U}_{P} \mid D\left(y_{1}, \ldots, y_{n}\right) \neq 0\right\}$ intersecting $\mathscr{O}_{i-1}$ nontrivially. $t$ is analytic in $\mathscr{U}_{i}$. Any element $G$ in $K_{i}$ is of the form $G=b_{r-1} t^{r}+\cdots+b_{0}$ for some $b_{j}$ in $K_{i-1}$ and we can argue as above to conclude (i) and (ii) for $K_{i}$. Finally to see that the substitution of $\bar{y}_{1}(z), \ldots, \bar{y}_{n}(z)$ for $y_{1}, \ldots, y_{n}$ defines an isomorphism $\varphi$ of $K_{m-1}$ onto a differential field $\bar{K}$ of meromorphic functions, note that $d(\varphi(G)) / d z=\sum \varphi\left(\partial G / \partial y_{j}\right) \varphi\left(f_{j}\right)$. This latter formula is just the chain rule.

Note that in the course of the proof, we may have changed the determination of the $t_{j}$ (for example, by extending past a branch cut). This will not effect the function defining the liouvillian relation since there will always remain a nonempty open set on which all the functions will agree with their original determinations.

We can now prove (iii). Let $\varphi: K_{M-1} \rightarrow \bar{K}$ be the isomorphism described in (ii), where $K$ is a field of functions meromorphic in $\mathscr{O}$ and let $M=K_{m-1}(t)$ where either $t$ is algebraic over $K_{m-1}$ or $t$ is transcendental over $K_{m-1}$ and $\partial t / \partial y_{j} \in K_{m-1}$ for $j=1, \ldots, m$ or $\partial t / \partial y_{j} / t \in K_{m-1}$ for $j=1, \ldots, m$. We first note that, under our assumption of minimality, $t$ cannot be algebraic over $K_{m-1}$. If it were, let $F \in M$ be the element defining the liouvillian relation and let $P(Y)=Y^{r}+a_{r-1} Y^{r-1}+\cdots+a_{0}$ be the minimal polynomial of $F$ over $K_{m-1}$. Since $a_{0} \neq 0$ in $K_{m-1}, a_{0}$ is analytic and nonzero on a dense open subset of $\mathscr{G}$. Since $P(F)=0$ and $\left.F\right|_{\mathscr{G}} \equiv 0$, we have $\left.a_{0}\right|_{\mathscr{G}} \equiv 0$, a 
contradiction. Therefore $t$ is transcendental over $K_{m-1}$ and we must deal with two cases. First assume that $\partial t / \partial y_{j} \in K_{m-1}$ for $j=1, \ldots, m-1$. We may assume that $F \in K_{m-1}[t]$. We can clearly extend $\varphi$ to a homomorphism on $K_{m-1}[t]$. Since $\varphi(F)=0$, we have that $\varphi(t)$ is algebraic over $\bar{K}$. Therefore, the Corollary to Theorem 1 of [ROS76] implies that there is an element $u$ of $\bar{K}$ such that $\varphi(t)-u$ is a constant. Let $U \in K_{m-1}$ satisfy $\varphi(U)=u$. We then have that $0=d(\varphi(t-U)) / d z=\sum \varphi\left(\partial(t-U) / \partial y_{j}\right) \varphi\left(f_{j}\right)=\varphi\left(\sum \partial(t-U) / \partial y_{j} \cdot f_{j}\right)$. Let $W=t-U$. Since $\varphi$ is an isomorphism on $K_{m-1}, D W \in K_{m-1}$ and $\varphi(D W)=0$, we have that $D W=0$. If $\partial W / \partial y_{j}=0$ for all $j$, then $W$ would be in $\mathbb{C}$. This would imply that $t$ would be algebraic over $K_{m-1}$ a contradiction.

Now assume that $\partial t / \partial y_{j} / t \in K_{m-1}$ for $j=1, \ldots, n$. Note that the $\partial t / \partial y_{i} / t$ are analytic and nonzero on some dense open subset of $\mathscr{G}$, so $t$ will be nonzero on a dense open subset of $\mathscr{G}$. Therefore, $\varphi(t) \neq 0$. Again we can assume that $F \in K_{m-1}[t]$ and conclude that $\varphi(t)$ is algebraic over $\bar{K}$. By the Corollary of Theorem 1 of [ROS76], we can conclude that there exists an integer $r$ such that $\varphi\left(t^{r}\right) \in \bar{K}$. Let $U \in K_{m-1}$ satisfy $\varphi\left(t^{r} / U\right)=1$ and let $W=t^{r} / U$. One can see that $D W / W \in K_{n-1}$ and $\varphi(D W / W)=0$, so $D W=0$. If $\partial W / \partial y_{j}=0$ for $j=1, \ldots, n$, then $W$ would be a constant and $t$ would be algebraic over $K_{m-1}$, a contradiction.

Note that the above proposition begins with an analytic hypothesis (the existence of a liouvillian relation) and ends with an algebraic conclusion (the existence of a liouvillian extension $M$ of $k$ and an element $W \in E$ such that $D W=0$ while $\partial W / \partial y_{j} \neq 0$ for some $j$ ). In what follows, we start with this latter statement as our hypotheses and use algebraic considerations to prove our theorems. The conclusions in the above result can be restated in the following way. We start with a differential field $(k, \Delta)$, where $\Delta=\left\{\delta_{i}\right\}$ is a set of commuting derivations and we let $D$ be some $k$-linear combination of a finite subset of $\Delta$. We concluded that there is liouvillian extension $(M, \Delta)$ of $(k, \Delta)$ such that $C(k, \Delta)=C(M, \Delta)$ and such that there is a $W$ in $M$ such that $D W=0$ while $\delta W \neq 0$ for some $\delta \in \Delta$. This latter condition can be restated as $C(M, \Delta)$ is a proper subset of $C(M,\{\Delta\})$. The next proposition starts with this as the hypothesis and draws some algebraic conclusions. It is our main technical tool and says that if there exists a liouvillian first integral, then there exist liouvillian functions $u_{1}\left(y_{1}, \ldots, y_{n}\right), \ldots, u_{n}\left(y_{1}, \ldots, y_{n}\right)$ such that $F\left(y_{1}, \ldots, y_{n}\right)=\int u_{1} d y_{1}+\cdots+u_{n} d y_{n}$ is a liouvillian first integral. The usefulness of the proposition lies in the fact that we may take the $u_{i}$ to lie in a liouvillian extension $N$ of $k$ satisfying $C(k, \Delta)=C(N, \Delta)=C(N,\{D\})$ and that $\delta_{i} u_{j}=\delta_{j} u_{i}$. The condition on the constants and the latter integrability conditions will be the key to the results of $\S \S 3$ and 4 .

Proposition 2.2. Let $(M, \Delta)$ be a liouvillian extension of the differential field $(k, \Delta)$ with $C(M, \Delta)=C(k, \Delta)$. Let $D=f_{1} \delta_{1}+\cdots+f_{n} \delta_{n}$ with $f_{i} \in k$, $\delta_{i} \in \Delta$, and assume that $C(k, \Delta)=C(k,\{D\})$. If $C(M, \Delta)$ is a proper subset of $C(M,\{D\})$, then there exists a liouvillian extension $(N, \Delta)$ of $(k, \Delta)$ such that

(i) $C(k, \Delta)=C(N, \Delta)=C(N,\{D\})$,

(ii) there exist $u_{i} \in N$, not all zero, such that $f_{1} u_{1}+\cdots+f_{n} u_{n}=0$ and $\delta_{i} u_{j}=\delta_{j} u_{i}$ for $1 \leq i, j \leq n$. 
Proof. Let $(M, \Delta)$ be a liouvillian extension of $(k, \Delta)$ of smallest transcendence degree over $k$ such that the hypotheses of the proposition are satisfied. Note that $M$ is not algebraic over $k$. If it were, then there would exist an element $u$, algebraic over $k$ such that $D u=0$, while $\delta u \neq 0$ for some $\delta \in \Delta$. We would then have, by Lemma 5.2 of [KAP57], that $u$ is algebraic over $C(k,\{D\})$. Since $C(k,\{D\})=C(k, \Delta)$, we would have $\delta u=0$ for all $\delta \in \Delta$, a contradiction. Therefore $M$ is algebraic over $N(t)$, where $N$ is a liouvillian extension of $k, t$ is transcendental over $N$, and either $\delta t \in N$ for all $\delta \in \Delta$ or $\delta t / t \in N$ for all $\delta \in \Delta$. We may further assume that $N$ is algebraically closed in $M$. Let $u$ be an element of $M$ such that $D u=0$ and $\delta u \neq 0$ for some $\delta \in \Delta . \quad u$ is the root of an irreducible polynomial $Y^{r}+b_{r-1} Y^{r-1}+\cdots+b_{0}$ with $b_{i} \in N(t)$. If $\delta b_{i}=0$ for all $\delta \in \Delta$ and $1 \leq i \leq r-1$, then $\delta u=0$ for all $\delta \in \Delta$. Similarly if $D b_{i} \neq 0$ for some $i$, we would have $D u \neq 0$. Therefore we may assume that there exists an element $u \in N(t)$ such that $D u=0$ and $\delta u \neq 0$ for some $\delta \in \Delta$. Note that by minimality $C(N,\{D\})=C(N, \Delta)=C(k, \Delta)$.

Assume that $\delta t \in N$ for all $\delta \in \Delta$. This implies that $D t \in N$. Since there exists a $u \in N(t)$ such that $D u=0$, we have by Proposition 1.2 of [RISCH69] that there are elements $\alpha \in N$ and $c \in N(t)$ such that $D c=0$ and $t=\alpha+c$. Note that $\delta c=\delta t-\delta \alpha \in N$ for all $\delta \in \Delta$. Furthermore if $\delta c=0$ for all $\delta \in \Delta$, then $c \in N$. Since $t \notin N$, we must have $\delta c \neq 0$ for some $\delta \in \Delta$. Let $u_{i}=\delta_{i} c$. We then have $\sum f_{i} u_{i}=\sum f_{i} \delta_{i} c=D c=0$. Since the $\delta_{i}$ commute, we have $\delta_{j} u_{i}=\delta_{j}\left(\delta_{i} c\right)=\delta_{i}\left(\delta_{j} c\right)=\delta_{i} u_{j}$.

Now assume that $\delta t / t \in N$ for all $\delta \in \Delta$. We then have that $D t / t \in N$. Since there is an element $u \in N(t), u \notin N$, such that $D u=0$, Proposition 1.2 of [RISCH69] implies that there are $\alpha \in N, c \in N(t)$ such that $D c=0$ and a nonzero integer $n$ such that $t^{n}=c \alpha$. Note that $\delta c / c=n \delta t / t-\delta \alpha / \alpha \in \nu$ for all $\delta \in \Delta$. Furthermore, if $\delta c=0$ for all $\delta \in \Delta$, then $c$ would lie in $N$, contradicting the fact that $t \notin N$. Let $u_{i}=\delta_{i} c / c$. We then have $\sum f_{i} u_{i}=0$ and $\delta_{i} u_{j}=\delta_{j} u_{i}$.

\section{Planar Vector fields}

To prove Theorem 1 and its Corollary we will use the results of $\S 2$ when $n=2$. We will use the variables $x$ and $y$ instead of $y_{1}$ and $y_{2}$ and let $k=\mathbb{C}(x, y)$. We will be able to deduce Theorem 1 from the following purely algebraic results.

Lemma 3.1. Let $K$ be a differential field of characteristic zero with derivation $D$ and $K(t)$ a differential extension with the same field of constants such that $D t \in K$. For any $a, b$ in $K$, any solution of

$$
D U+a U=b,
$$

in $K(t)$ is of the form $U_{0}+U_{1} t$ for some $U_{0}, U_{1}$ in $K$.

Proof. By comparing partial fraction decompositions of $a, b$, and $U$, we see that any solution of (4) is in $K[t]$. If $U_{0}+U_{1} t+\cdots+U_{n} t^{n}$ is such a solution with $n \geq 2$, then, equating powers of $t$, we have $D U_{n}+a U_{n}=0$ and $n U_{n} D t+$ $D U_{n-1}+a U_{n-1}=0$. Multiplying this latter equation by $U_{n}^{-1}$ allows us to conclude that $D\left(U_{n}^{-1} U_{n-1}\right)=-n D t$. This implies that $t \in K$, a contradiction. 
Lemma 3.2. Let $\left(k,\left\{\delta_{1}, \delta_{2}\right\}\right)$ be a differential field of characteristic zero, $N$ a liouvillian extension of $k$ with $C\left(N,\left\{\delta_{1}, \delta_{2}\right\}\right)=C\left(k,\left\{\delta_{1}, \delta_{2}\right\}\right)$ and $P$ and $Q$ elements of $N$. Assume that

(i) $C\left(N,\left\{\delta_{1}, \delta_{2}\right\}\right)=C(N,\{D\})$ where $D=P \delta_{1}+Q \delta_{2}$;

(ii) $P U+Q V=0, \delta_{2} U=\delta_{1} V$ has only the trivial solution $U=V=0$ in $N$

(iii) $P U+Q V=-\left(\delta_{1} P+\delta_{2} Q\right), \delta_{2} U=\delta_{1} V$ has a solution in $N$.

Then there exists $U_{0}, V_{0}$ in $k$ satisfying $P U_{0}+Q V_{0}=-\left(\delta_{1} P+\delta_{2} Q\right)$ and $\delta_{2} U_{0}=\delta_{1} V_{0}$.

Proof. We proceed by induction on the transcendence degree of $N$ over $k$. We may therefore assume (by taking traces if necessary) that there exist $U$ and $V$ in $\hat{k}(t)$ satisfying the equations in (iii) where $\hat{k}$ is an algebraic extension of $k$, and either $\delta_{1} t, \delta_{2} t \in \hat{k}$ or $\delta_{1} t / t, \delta_{2} t / t \in \hat{k}$. We will first show that we can find $U_{0}$ and $V_{0}$ in $\hat{k}$ satisfying the equations in (iii).

First assume that $\delta_{1} t / t$ and $\delta t / t$ are in $\hat{k}$. Expanding $U$ and $V$ in decreasing powers of $t$, we have $U=U_{n} t^{n}+U_{n-1} t^{n-1}+\cdots$ and $V=V_{m} t^{m}+$ $V_{m-1} t^{m-1}+\cdots$, with the $U_{i}$ and $V_{i}$ in $\hat{k}$. Comparing powers of $t$, we can conclude that $P U_{0}+Q V_{0}=-\left(\delta_{1} P+\delta_{2} Q\right)$. Comparing the coefficient of $t^{0}$ in $\delta_{2} U$ and $\delta_{1} V$ we see that $\delta_{2} U_{0}=\delta_{1} V_{0}$.

Now assume that $\delta_{1} t$ and $\delta_{2} t$ are in $\hat{k}$. We therefore have $D t \in \hat{k}$. Applying $\delta_{1}$ to $P U+Q V=-\left(\delta_{1} P+\delta_{2} Q\right)$ allows us to conclude that $D U+a U=b$ for some $a, b$ in $\hat{k}$. Lemma 3.1 implies that $U=U_{0}+U_{1} t$ for some $U_{0}, U_{1} \in \hat{k}$. Similarly, we can show that $V=V_{0}+V_{1} t$ for some $V_{0}, V_{1}$ in $\hat{k}$. Comparing powers of $t$ in the equations in (iii), we conclude that $P U_{1}+Q V_{1}=0$ and $\delta_{2} U_{1}=\delta_{1} V_{1}$, so by (ii), $U_{1}=V_{1}=0$. Therefore $U=U_{0}$ and $V=V_{0}$ are in $\hat{k}$.

Given a solution of the equations in (iii) in $\hat{k}$, we can take traces with respect to $k$ and conclude that there exist $U_{0}$ and $V_{0}$ in $k$ satisfying the conclusion of the proposition.

Proposition 3.3. Let $\left(k,\left\{\delta_{1}, \delta_{2}\right\}\right)$ be a differential field of characteristic zero and let $P, Q \in k$. Let $D=P \delta_{1}+Q \delta_{2}$ and assume that $C\left(k,\left\{\delta_{1}, \delta_{2}\right\}\right)=$ $C(k,\{D\})$. If there exists a liouvillian extension $\left(M,\left\{\delta_{1}, \delta_{2}\right\}\right)$ of $k$ such that $C\left(k,\left\{\delta_{1}, \delta_{2}\right\}\right)=C\left(M,\left\{\delta_{1}, \delta_{2}\right\}\right)$ and such that $C\left(M,\left\{\delta_{1}, \delta_{2}\right\}\right)$ is a proper subset of $C(M,\{D\})$, then there exist elements $U$ and $V$ in $k$ such that:

$$
\begin{aligned}
P U+Q V & =-\left(\delta_{1} P+\delta_{2} Q\right), \\
\delta_{2} U & =\delta_{1} V .
\end{aligned}
$$

Proof. By Proposition 2.2, there exists a liouvillian extension $\left(N,\left\{\delta_{1}, \delta_{2}\right\}\right)$ of $\left(k,\left\{\delta_{1}, \delta_{2}\right\}\right)$ such that $C\left(k,\left\{\delta_{1}, \delta_{2}\right\}\right)=C\left(N,\left\{\delta_{1}, \delta_{2}\right\}\right)=C(N,\{D\})$ and nonzero elements $u_{1}$ and $u_{2}$ in $N$ such that $P u_{1}+Q u_{2}=0$ and $\delta_{2} u_{1}=\delta_{1} u_{2}$. Furthermore we shall assume that $N$ has the smallest transcendence degree over $k$ of all such extensions. Letting $R=u_{1} / Q$, we see that $D R=-\left(\delta_{1} P+\delta_{2} Q\right) R$. Let $U=\delta_{1} R / R$ and $V=\delta_{2} R / R$. If $N$ is algebraic over $k$, then replacing $U$ and $V$ by a constant multiple of their traces, allows us to reach the conclusion of the proposition. Therefore assume that $N$ is algebraic over $K(t)$, where either $\delta_{1} t, \delta_{2} t \in K$ or $\delta_{1} t / t, \delta_{2} t / t \in K, t$ is transcendental over $K$, and $K$ is algebraic closed in $N$. We will show that $U$ and $V$ are in $K$. 
Assume that $\delta_{1} t, \delta_{2} t \in K$. We then have that $D t \in K$. Since $D R / R \in K$, Theorem 2 of [ROS76] implies that $R \in K$. Therefore $U=\delta_{1} R / R$ and $V=\delta_{2} R / R$ are in $K$. Now assume that $\delta_{1} t / t, \delta_{2} t / t \in K$. Since $D t / t$ and $D R / R \in K$, Theorem 2 of [ROS76] implies that $R^{n} t^{m} \in K$ for some integers $n$ and $m, n \neq 0$. Therefore $U=\delta_{1} R / R$ and $V=\delta_{2} R / R$ are in $K$.

By the minimality in the choice of $K, K$ satisfies the hypotheses of Lemma 3.2. We therefore can conclude that there exists $U$ and $V$ in $K$ satisfying (5) above.

Proof of Theorem 1. The hypotheses of Theorem 1 imply that the solution $(\bar{x}(z), \bar{y}(z))$ satisfies a liouvillian relation. As we have noted following the definition of the basic assumption, if $\bar{x}(z)$ and $\bar{y}(z)$ are not algebraically dependent over $\mathbb{C}$ (that is, there is no nonzero polynomial $G$ such that $G(\bar{x}(z), \bar{y}(z)$ ) $=0)$, then $(\bar{x}(z), \bar{y}(z))$ satisfies the basic assumption. Therefore, Proposition 2.1 implies that (1) has a liouvillian first integral. In particular the hypotheses of Proposition 3.3 are satisfied where $k=\mathbb{C}(x, y)$ and $\delta_{1}=\partial / \partial x$ and $\delta_{2}=\partial / \partial y$. We can conclude that there exist $U$ and $V$ in $k=\mathbb{C}(x, y)$ such that (5) holds. Letting

$$
R(x, y)=\exp \left[\int_{\left(x_{0}, y_{0}\right)}^{(x, y)} U(x, y) d x+V(x, y) d y\right]
$$

we see that $\partial(R P) / \partial x=-\partial(R Q) / \partial y$ so the conclusion of Theorem 1 is valid.

Proof of the corollary to Theorem 1. As we have already noted, the existence of an integrating factor of the form (2) guarantees a liouvillian first integral.

Conversely, assume that (1) has a liouvillian first integral $F$ analytic on some open nonempty subset $\mathscr{U}$ of $\mathbb{C}^{2}$. If for any solution $(x(z), y(z))$ passing through this open set there is a polynomial $G$ such that $G(x(z), y(z))=0$, then there exist an infinite number of algebraic solution curves. Darboux's Theorem (see the appendix) implies that there is a first integral of the form $F(X, Y)=\prod_{i=1}^{n} G_{i}^{n_{i}}$, where the $G_{i}$ are polynomials in $x$ and $y$ with constant coefficients. $F$ will then satisfy $P \partial F / \partial x+Q \partial F / \partial y=0$, so $R=(\partial F / \partial x) / Q$ will be an integrating factor. Since $R \in C(x, y)$, it clearly can be written in the form (2). Now assume that some solution curve in $\mathscr{U}$ of $(1)$ is not algebraic and that (1) has a liouvillian first integral. The conclusion of the corollary then follows from Theorem 1 .

We now turn to the problem of finding liouvillian first integrals. Theorem 1 tells us what to look for to find liouvillian first integrals for equations (1), but we are far from a decision procedure. We are even unable to give a complete procedure to decide if (1) has a rational first integral, i.e., if there exists a $W \in \mathbb{C}(x, y)$ such that $D W=0$. In [PRSI83] we reduced this latter problem to the following problem:

Give $D$ as above, effectively find an integer $N$ so that if $G$ is irreducible in $\mathbb{C}[x, y]$ and $G$ divides $D G$, then $\operatorname{deg} G<N$.

We know from the corollary to Darboux's Theorem in the appendix that such an $N$ exists. In [PRSI83], we also reduced the problem of finding elementary first integrals of (1) (i.e., first integrals expressible in terms of exponentials, logarithms and algebraic functions), to the above problem (6). We are unable to do this for liouvillian first integrals. We are able to make the following 
reduction. If $R$ is of the form (2), then (by applying $\partial / \partial x$ to (5)) $U$ will satisfy

$$
D U+Q(P / Q)_{x} U=-Q\left[\left(P_{x}+Q_{y}\right) / Q\right]_{x},
$$

where the subscripts denote partial differentiation. A similar equation for $V$ can be found. The problem of finding a liouvillian first integral for (1) can be reduced to algorithmically solving the following problem.

$$
\begin{aligned}
& \text { Given } D \text { as above and } a, b \in \mathbb{C}(x, y) \text {, find all solutions } U \in \\
& \mathbb{C}(x, y) \text { of } D U+a U=b .
\end{aligned}
$$

The special case when $b=0$ arose in [PRSI83] and we were able to reduce (8) in that special case to the problem (6). We are unable to make a similar reduction in the general case. Of course, under special assumptions (7) can be solved. For example if $\left(P_{x}+Q_{y}\right) / P$ is independent of $y$, then we may let $U=\left(P_{x}+Q_{y}\right) / Q$ and $V=0$ to find an integrating factor (cf. [BODI77, p. 43]). When $P=1$ and $Q=(d f(x) / d x) / f(x)$ or $Q=y d f(x) / d x$ with $f(x) \in \mathbb{C}(x)$, an algorithm to solve (8) is given in [RISCH69].

\section{LINEAR DIFFERENTIAL EQUATIONS}

As in $§ 3$, we will combine purely algebraic considerations with Propositions 2.1 and 2.2 to prove Theorem 2 and its corollary. Let $k_{0}$ be an ordinary differential field with derivation $D$ and let $L(y)=D^{(n)} y+a_{n-1} D^{(n-1)} y+\cdots+$ $a_{0} y=0$ be a linear differential equation with coefficients in $k_{0}$. We formally construct a differential field $k$ with $n+1$ derivations $\Delta=\left\{\delta_{-1}, \delta_{0}, \ldots, \delta_{n-1}\right\}$ in the following manner. Let $k=k_{0}\left(y_{0}, y_{1}, \ldots, y_{n-1}\right)$ where we consider $y_{0}, \ldots, y_{n-1}$ as algebraically independent indeterminates. We let $\delta_{-1}$ agree with $D$ on $k_{0}$ and extend it to $k$ by setting $\delta_{-1}\left(y_{i}\right)=0$ for $i=0, \ldots, n-1$. For $j=0, \ldots, n-1$, we let $\delta_{j}$ be zero on $k_{0}$ and $\delta_{j}\left(y_{i}\right)=1$ if $i=j$ and 0 otherwise. We extend $D$ to $k$ (and use the same letter to denote this extension) by setting $D=\delta_{-1}+y_{1} \delta_{0}+\cdots+y_{n-1} \delta_{n-2}+\left(-a_{n-1} y_{n-1}-\cdots-a_{0} y_{0}\right) \delta_{n-1}$. Note that $D^{(n)} y_{0}+a_{n-1} D^{(n-1)} y_{0}+\cdots+a_{0} y_{0}=0$, so we have an element $y_{0}$ in $k$ satisfying $L(y)=0$. Theorem 2 follows from Proposition 4.2 below which depends in Proposition 2.2 and the following lemma. Recall that the adjoint $L^{*}(z)$ of $L(y)$ is defined as [POOLE60], p. 38.

$$
L^{*}(z)=(-1)^{n} D^{(n)} z+(-1)^{n-1} D^{(n-1)}\left(a_{n-1} z\right)+\cdots-D\left(a_{1} z\right)+a_{n} z .
$$

Lemma 4.1. Let $(K, \Delta)$ be as above and let $(F, \Delta)$ be an extension of $(K, \Delta)$ containing elements $u_{i},-1 \leq i \leq n-1$, such that

(i) $u_{-1}+y_{0} u_{0}+\cdots+y_{n-1} u_{n-2}+\left(-a_{n-1} y_{n-1}-\cdots-a_{0} y_{0}\right) u_{n-1}=0$,

(ii) $\delta_{i} u_{j}=\delta_{j} u_{i},-1 \leq i, j \leq n-1$.

Then $u_{n-1}$ satisfies $L^{*}\left(u_{n-1}\right)=0$ where $L^{*}(z)$ is the adjoint of $L(y)$.

Proof. Let $A$ be the $n \times n$ matrix

$$
\left[\begin{array}{ccccc}
0 & 1 & 0 & \cdots & 0 \\
0 & 0 & 1 & \cdots & 0 \\
& & \vdots & & \\
0 & 0 & & \cdots & 1 \\
-a_{0} & -a_{1} & & \cdots & -a_{n-1}
\end{array}\right],
$$


and let $B$ be the $(n+1) \times(n+1)$ matrix

$$
\left[\begin{array}{ll}
1 & 0 \\
0 & A
\end{array}\right] \text {. }
$$

Let $U$ and $Y$ be the $(n+1) \times 1$ column vectors $\left(u_{-1}, \ldots, u_{n-1}\right)^{T}$ and $\left(1, y_{0}, \ldots, y_{n-1}\right)^{T}$. Note that $D\left(\left(y_{0}, y_{1}, \ldots, y_{n-1}\right)^{T}\right)=A\left(y_{0}, \ldots, y_{n-1}\right)^{T}$ and that this is just the matrix form of $L(y)=0$. By (i), we have $\langle B Y, U\rangle=0$, where $\langle$,$\rangle is the usual inner product. Applying \delta_{i}$ to this latter equation we have $0=\delta_{i}(\langle B Y, U\rangle)=\left\langle\delta_{i}(B Y), U\right\rangle+\left\langle B Y, \delta_{i}(U)\right\rangle$. We shall calculate each of these terms separately. For $i=0, \ldots, n-1, \delta_{i}$ annihilates the entries of $B$, so we have $\delta_{i}(B Y)=B\left(\delta_{i} Y\right)$. By (ii), we have that $\delta_{i}(U)=$ $\left(\delta_{-1} u_{i}, \ldots, \delta_{n-1} u_{i}\right)^{T}$. Therefore, for $i=0, \ldots, n-1,0=\delta_{i}(\langle B Y, U\rangle)=$ $\left\langle B\left(\delta_{i} Y\right), U\right\rangle+\left\langle B Y,\left(\delta_{-1} u_{i}, \ldots, \delta_{n-1} u_{i}\right)^{T}\right\rangle=\left\langle\delta_{i} Y, B^{T} U\right\rangle+D u_{i}=\left(B^{T} U\right)_{i+2}+$ $D u_{i}$. We therefore have the relation

$$
D\left[\begin{array}{c}
u_{0} \\
\vdots \\
u_{n-1}
\end{array}\right]=-A^{T}\left[\begin{array}{c}
u_{0} \\
\vdots \\
u_{n-1}
\end{array}\right]
$$

From this latter equation one can easily calculate that $L^{*}\left(u_{n-1}\right)=0$.

Propositon 4.2. Let $(k, \Delta)$ be as above with $a_{1}=0$ and $n=2$. Assume that $C(k, \Delta)=C\left(k_{0}, \Delta\right)$. If there is a liouvillian extension $(M, \Delta)$ of $(k, \Delta)$ such that $C(k, \Delta)=C(M, \Delta) \neq C(M,\{D\})$, then all solutions of $L(y)=0$ are liouvillian over $k$.

Proof. The assumptions imply that $L(y)=y^{\prime \prime}+a_{0} y$ and therefore that $L^{*}(z)=$ $z^{\prime \prime}+a_{0} z$. Proposition 2.2 implies that there is a liouvillian extension $(N, \Delta)$ of $(k, \Delta)$ such that $C(N, \Delta)=C(N,\{D\})=C(k, \Delta)$ and elements $u_{-1}, u_{0}, u_{1}$ in $N$, not all zero, such that

$$
u_{-1}+u_{0} y_{1}+u_{1}\left(-a_{0} y_{0}\right)=0,
$$

and $\delta_{i} u_{j}=\delta_{j} u_{i}$ for $-1 \leq i, j \leq 1$. Lemma 4.1 implies that $D^{2} u_{1}+a_{0} u_{1}=0$. We shall show that $u_{1}$ is algebraic over $K$. Proceeding by induction on the transcendence degree of $N$ over $k$, we may assume that $N$ is algebraic over $\bar{k}(t)$ where $\delta_{i} t \in \bar{k}$ or $\delta_{i} t / t \in \bar{k}$ for $i=-1,0$ or 1 and $\bar{k}$ is algebraic over $k$.

We first assume that $\delta_{i} t / t \in \bar{k}$ for $i=-1,0$ or 1 . Note that since $a_{1}=0$, we have that the Wronskian determinant of any two solutions of $L(y)=0$ is constant. Therefore $D\left(u_{1} / y_{0}\right)=c / y_{0}^{2} \in K$. Since $D t / t \in K$, Theorem 2 of [ROS76] implies that $u_{1}$ is algebraic over $k$.

Now assume that $\delta_{i} t \in \bar{k}$ for $i=-1,0$ and 1. Since $D\left(u_{1} / y_{0}\right) \in k$, Theorem 2 of [ROS76] implies that there are constants $c_{1}$ and $c_{2}$, not both zero, such that $c_{1}\left(u_{1} / y_{0}\right)+c_{2} t \in \bar{K}$. If $c_{2}=0$, then $u_{1} \in \bar{k}$, so we may assume that $c_{2} \neq 0$ (and therefore that $c_{1} \neq 0$ ). We shall show how this leads to a contradiction. Replacing $t$ by $c t+\alpha$ for suitable $\alpha \in \bar{k}$ and constant $c$, we may assume that $D t=1 / y_{0}^{2}$. Therefore there exist $v_{-1}, v_{0}$, and $v_{1}$ in $\bar{k}$ such that $\delta_{i} v_{j}=\delta_{j} v_{i},-1 \leq i, j \leq 1$, and

$$
v_{-1}+y_{1} v_{0}-a_{0} y_{0} v_{1}=\frac{1}{y_{0}^{2}} \text {. }
$$


Applying $\delta_{1}$ to (10), we have

$$
\begin{aligned}
0 & =\delta_{1} v_{-1}+v_{0}+y_{1} \delta_{1} v_{0}-a_{0} y_{0} \delta_{1} v_{1} \\
& =\delta_{-1} v_{1}+v_{0}+y_{0} \delta_{0} v_{1}-a_{0} \delta_{1} v_{1} \\
& =D v_{1}+v_{0} .
\end{aligned}
$$

Therefore $D v_{1}=-v_{0}$. Applying $\delta_{0}$ to (10), we similarly conclude that $D v_{0}=$ $a_{0} v_{1}-2 / y_{0}^{3}$. Therefore $D^{2} v_{1}+a_{0} v_{1}=2 / y_{0}^{3}$. Letting $w=v_{1} / y_{0} \in \bar{k}$, we find that $\left(\frac{1}{2} D w y_{0}^{2}\right)^{\prime}=1 / y_{0}^{2}$. Therefore $\frac{1}{2} D w y_{0}^{2}$ and $t$ differ by a constant. Since $\frac{1}{2} D w y_{0}^{2} \in \bar{k}$, we have a contradiction.

We therefore conclude that $u_{1}$ is algebraic over $k$. If $u_{1}$ and $y_{0}$ are linearly independent then $\bar{k}$ contains the Picard-Vessiot extension of $k_{0}$ corresponding to $L(y)=0$. Since the transcendence degree of $\bar{k}$ over $k_{0}$ is 2 , we can conclude that the connected component of the identity of the galois group of $L(y)=0$ is solvable [KOL73, p. 373], and so all solutions of $L(y)=0$ are liouvillian. To complete the proof, we shall show that the assumption that $u_{1}$ and $y_{0}$ are linearly dependent leads to a contradiction. We first note that by applying $\delta_{1}$ to (9), we can conclude that $D u_{1}=-u_{0}$. Therefore if $u_{1}=c y_{0}$ for some constant $c$, we have (again from (9)) that

$$
u_{-1}-c\left(D y_{0}\right)^{2}-a_{0} c y_{0}^{2}=0 .
$$

Since $\delta_{0} u_{-1}=\delta_{-1} u_{0}=\delta_{-1}(c D y)=0$ and $\delta_{1} u_{-1}=\delta_{-1} u_{1}=\delta_{-1}\left(c y_{0}\right)=0$, we have that $u_{-1}$ is algebraic over $C\left(k,\left\{\delta_{0}, \delta_{1}\right\}\right)=k_{0}$. We must have $c \neq 0$ (otherwise $u_{-1}=u_{0}=u_{1}=0$ ), so (11) implies that $y_{0}$ and $D y_{0}=y_{1}$ are algebraically dependent over $k_{0}$, a contradiction.

Proof of Theorem 2. Let $k_{0}$ be a liouvillian extension of the complex numbers containing $p$ and $q$. If necessary, we can adjoin $e^{\int p}$ to $k_{0}$ and replace $y$ by $e^{\int p} y$ in $L(y)$ to assume that $L(y)=y^{\prime \prime}+a_{0} y$. This modification on $L(y)$ does not affect the validity of our hypotheses. If $\bar{y}$ and $d \bar{y} / d z$ are algebraically dependent over $k_{0}$, then $L(y)=0$ has a solution in an extension of $k_{0}$ of transcendence degree 1 over $k_{0}$. Corollary 3 of [SIN76] implies that $L(y)=0$ will have a nonzero liouvillian solution. Since $L(y)$ has order 2 , all solutions are then liouvillian.

We may therefore assume that $\bar{y}$ and $d \bar{y} / d z$ are algebraically independent over $k_{0}$. Note that $y_{1}=\bar{y}, y_{2}=d \bar{y} / d z, y_{3}=z$ satisfy

$$
\frac{d y_{1}}{d z}=y_{2}, \quad \frac{d y_{2}}{d z}=-a_{0} y_{1}, \quad \frac{d y_{3}}{d z}=1 .
$$

We may think of $a_{0}$ as a function of $y_{3}$. Let $k_{0}$ be the differential extension of $\mathbb{C}\left(y_{3}\right)$ generated by $a_{0}$ and let $k=k_{0}\left(y_{1}, y_{2}\right)$. As we have noted following the definition of the basic assumption, the algebraic independence of $\bar{y}_{1}$ and $\bar{y}_{2}$ implies the basic assumption. Proposition 2.1 implies that the hypotheses of Proposition 4.2 are satisfied. Therefore Proposition 4.2 implies that Theorem 2 is true.

Proof of the corollary to Theorem 2. The first part of the corollary is an immediate consequence of Theorem 2. To see that the second part holds, let $y(z)$ be a nonzero liouvillian solution of $L(y)=0$. Letting $u(z)=y^{\prime}(z) / y(z)$, we can write $L(y)=L_{1}\left(L_{2}(y)\right)$, where $L_{2}(y)=y^{\prime}-u(z) y$ and $L_{1}(y)=y^{\prime}-v(z) y$ for 
some liouvillian function $v$. Any solution of $L(y)=0$ satisfies $L_{2}(y)=c e^{\int v}$ for some constant $c$. Therefore, $F\left(z, y, y^{\prime}\right)=e^{-\int v}\left(y^{\prime}-u(x) y\right)$ is a liouvillian first integral.

The corollary of Theorem 2 implies that the problem of finding liouvillian first integrals of a second order homogeneous linear differential equation is equivalent to finding nonzero liouvillian solutions of this equation. Kovacic [KOV86] has given an algorithm that solves this latter problem.

\section{APPENDIX}

We give here a proof of Darboux's Theorem that was alluded to in the proof of the corollary to Theorem 1. The original (weaker) version is described in [INCE56, pp. 29-32]. The present result occurs (in a stronger form) in [JOU79, Theorem 3.3, p. 102 and Lemma 3.53, p. 112]. Our proof, although ultimately in a spirit similar to [JOU79], only relies on [ROS76] and elementary facts about the resultant. We hope this will make it more accessible to workers in the field of integration in finite terms.

We first prove the following lemma. Let $P$ and $Q$ be polynomials in $\mathbb{C}[x, y]$ and consider the derivation $D=P \partial / \partial x+Q \partial / \partial y$. We say that $\left(x_{0}, y_{0}\right) \in \mathbb{C}^{2}$ is a nonsingular point of $D$ if $P\left(x_{0}, y_{0}\right) \neq 0$ or $Q\left(x_{0}, y_{0}\right) \neq 0$.

Lemma A.1. Let $D$ be as above and $\left(x_{0}, y_{0}\right)$ a nonsingular point of $D$. Let $H_{1}$ and $H_{2}$ be polynomials such that $H_{1}$ divides $D H_{1}, H_{2}$ divides $D H_{2}, H_{1}\left(x_{0}, y_{0}\right)$ $=H_{2}\left(x_{0}, y_{0}\right)=0$ and $H_{1}$ irreducible. Then $H_{1}$ divides $H_{2}$.

Proof. Let us assume that $P\left(x_{0}, y_{0}\right) \neq 0$. The case $Q\left(x_{0}, y_{0}\right) \neq 0$ is similar. Let $R(x)$ be the resultant of $H_{1}$ and $H_{2}$ with respect to $y$. We may write $R(x)=A_{0} H_{1}+B_{0} H_{2}$ for some $A_{0}, B_{0} \in \mathbb{C}[x, y]$. Note $R\left(x_{0}\right)=0$. $D R=$ $P R^{\prime}=\left(D A_{0}\right) H_{1}+\left(D B_{0}\right) H_{2}+A_{0} D H_{1}+B_{0} D H_{2}=A_{1} H_{1}+B_{1} H_{2}$ for some $A_{1}, B_{1}$ in $\mathbb{C}[x, y]$, since $H_{1}$ divides $D H_{1}$ and $H_{2}$ divides $D H_{2}$. Since $P\left(x_{0}, y_{0}\right) \neq$ 0 , we have $R^{\prime}\left(x_{0}\right)=0$. Applying $D$ again, we get $(D P) R^{\prime}+P^{2} R^{\prime \prime}=A_{2} H_{1}+$ $B_{2} H_{2}$ for some $A_{2}, B_{2}$ in $\mathbb{C}[x, y]$. This equation implies that $R^{\prime \prime}\left(x_{0}\right)=0$. Continuing in this way we have $R^{(n)}\left(x_{0}\right)=0$ for all $n$, so $R(x) \equiv 0$. Therefore $H_{1}$ and $H_{2}$ have a common factor, so $H_{1}$ divides $H_{2}$.

One should think of this lemma as an algebraic counterpart of the uniqueness of solutions of differential equations. It implies that, at a nonsingular point, the vector field $P \partial / \partial x+Q \partial / \partial y$ has at most one solution that is an algebraic curve.

Darboux's Theorem. Let $P, Q \in \mathbb{C}[x, y]$ and let $D=P \partial / \partial x+Q \partial / \partial y$. If $G_{1}, \ldots, G_{m}$ are relatively prime irreducible polynomials in $\mathbb{C}[x, y]$ such that $G_{i}$ divides $D G_{i}$ for $i=1, \ldots, m$, then either $m<[d(d+1) / 2]+2$ where $d=\max (\operatorname{deg} P, \operatorname{deg} Q)$ or there exist integers $n_{i}$ not all zero such that $D w=0$ where $w=\prod_{i=1}^{m} G_{i}^{n_{i}}$. In the latter case, if $G$ is any irreducible polynomial such that $G$ divides $D G$, then either there exists $c_{1}, c_{2}$ in $\mathbb{C}$, not both zero such that $G$ divides $c_{1} \prod_{i \in I} G_{i}^{n_{i}}-c_{2} \prod_{j \in J} G_{j}^{-n_{j}}$ where $I=\left\{i \mid n_{i} \geq 0\right\}$ and $J=\left\{j \mid n_{j}<0\right\}$, or $G$ divides $\operatorname{GCD}(P, Q)$.

Proof. Let $G_{1}, \ldots, G_{m}$ be as above and assume that $m \geq[d(d+1) / 2]+2$. The polynomials $D G_{i} / G_{i}$ all have degree less than or equal to $d+\left(\operatorname{deg} G_{i}-1\right)$ $-\operatorname{deg} G_{i}=d-1$. The vector space of polynomials of degree at most $d-1$ has dimension $d(d+1) / 2$ so there exist $\mathbb{C}$-linearly independent vectors $\left(r_{i}\right)$, 
$\left(s_{i}\right) \in \mathbb{C}^{m}$ such that $\sum r_{i}\left(D G_{i} / G_{i}\right)=\sum s_{i}\left(D G_{i} / G_{i}\right)=0$. Let $k=\{w \in$ $\mathbb{C}(x, y) \mid D w=0\}$ and let $K=\mathbb{C}(x, y)$. We shall use the notation and results of $\S 1$ of [ROS76]. Let $\omega_{1}=\sum r_{i}\left(d G_{i} / G_{i}\right)$ and $\omega_{2}=\sum s_{i}\left(d G_{i} / G_{i}\right)$, where we consider $\omega_{1}, \omega_{2} \in \Omega_{K / k}$. Let $\bar{D}=(1 / P) D$. Note that $\bar{D}^{1}\left(\omega_{1}\right)=\bar{D}^{1}\left(\omega_{2}\right)=$ $\bar{D}^{1}(d x)=0$. Since tr. $\operatorname{deg}{ }_{k} K \leq 2, \operatorname{dim}_{K} \Omega_{K / k} \leq 2$. Proposition 6 of [ROS76] implies that there are $c_{1}, c_{2}, c_{3}$ in $k$ such that $0=c_{1} d x+c_{2} \omega_{1}+c_{3} \omega_{2}=$ $d\left(c_{1} x\right)+\sum\left(c_{2} r_{i}+c_{3} s_{i}\right)\left(d G_{i} / G_{i}\right)$. Let $t_{1}, \ldots, t_{n}$ be a basis for the $\mathbb{Q}$-vector space spanned by $\left\{c_{2} r_{i}+c_{3} s_{i}\right\}$ and let $c_{2} r_{i}+c_{3} s_{i}=(1 / N) \sum p_{i j} t_{j}$ where $p_{i j}$ are integers, not all $p_{i j}=0$. Let $F_{j}=\prod_{i=1}^{m} G_{i}^{p_{i j}}$. We then have $0=d\left(c_{1} x\right)+$ $\sum\left(t_{j} / N\right)\left(d F_{j} / F_{j}\right)$. Proposition 4 of [ROS76] then implies that $F_{1}, \ldots, F_{n}$ are algebraic over $k$. This implies that $\bar{D} F_{i}=D F_{i}=0$. Since some $p_{i j} \neq 0$, say $p_{11} \neq 0, w=F_{1}$ satisfies the conclusion of the first part of the theorem.

Now let $G$ be an irreducible polynomial such that $G$ divides $d G$ and let $F=\prod_{i \in I} G_{i}^{n_{i}}$ and $H=H_{j \in J} G_{j}^{n_{j}}$. We shall assume that $G$ does not divide $\operatorname{GCD}(P, Q)$ and show that there are $c_{1}, c_{2}$ in $\mathbb{C}$ such that $G$ divides $c_{1} F-$ $c_{2} H$. If we replace $D$ by $(1 / \operatorname{GCD}(P, Q)) D$, we can assume $\operatorname{GCD}(P, Q)=1$ and preserve the facts that $D w=0$ and $G$ divides $D G$. If $H=0$ implies that $G=0$, then $G$ divides $H$ and we have the final conclusion of the theorem. Therefore we may assume that $G=H=0$ has only a finite number of solutions. Furthermore, since $\operatorname{GCD}(P, Q)=1, P=Q=0$ has only a finite number of solutions. Therefore, there exists a point $\left(x_{0}, y_{0}\right)$ such that $G\left(x_{0}, y_{0}\right)=0$, $H\left(x_{0}, y_{0}\right) \neq 0$ and either $P\left(x_{0}, y_{0}\right) \neq 0$ or $Q\left(x_{0}, y_{0}\right) \neq 0$. Let $c_{1}=H\left(x_{0}, y_{0}\right)$ and $c_{2}=F\left(x_{0}, y_{0}\right)$ and let $S(x, y)=c_{1} F-c_{2} H$. Since $D(S / H)=0$, we have $H D S-S D H=0$. Since $S$ and $H$ have no common factors, $S$ divides $D S$. Applying Lemma A.1, allows us to conclude the $G$ divides $S$.

Corollary. Let $D$ be as above. Either there are at most a finite number of irreducible polynomials $G$ such that $G$ divides $D G$ or there is a nonzero rational function $W$ such that $D W=0$. In any case, there is an integer $N$, depending on $D$, such that if $G$ is an irreducible polynomial and $G$ divides $D G$, then $\operatorname{deg} f \leq N$.

Note that $N$ does not only depend on the degrees of $P$ and $Q$ but may also depend on the coefficients of $P$ and $Q$. For example, if $D=(n+1) x(\partial / \partial x)+$ $n y(\partial / \partial y)$, then for $G=x^{n}-y^{n+1}, G$ divides $D G$.

\section{REFERENCES}

[BODI77] W. E. Boyce and R. C. DiPrima, Elementary differential equations, Third ed., Wiley, New York, 1977

[INCE56] E. L. Ince, Ordinary differential equations, Dover, New York, 1956.

[JOU79] J. P. Jouanalou, Equations de Pfaff algebriques, Lecture Notes in Math., vol. 708, SpringerVerlag, Berlin and New York, 1979.

[KAP57] I. Kaplansky, An introduction to differential algebra, Hermann, Paris, 1957.

[KOL73] E. R. Kolchin, Differential algebra and algebraic groups, Academic Press, New York, 1973.

[KOV86] J. Kovacic, An algorithm for solving second order linear homogeneous differential equations, J. Symbolic Comput. 2 (1986).

[KRA82] S. G. Krantz, Function theory of several complex variables, Wiley, New York, 1982.

[LANG65] S. Lang, Algebra, Addison-Wesley, Reading, Mass., 1965. 
[POOLE60] E. G. C. Poole, Introduction to the theory of linear differential equations, Dover, New York, 1960.

[PRSI83] M. J. Prelle and M. F. Singer, Elementary first integrals of differential equations, Trans. Amer. Math. Soc. 279 (1983).

[RISCH69] R. H. Risch, The problem of integration in finite terms, Trans. Amer. Math. Soc. 139 (1969).

[RITT27] J. F. Ritt, On the integration in finite terms of linear differential equations of the second order, Bull Amer. Math. Soc. 33 (1927).

[RITT48] _ _ Integration in finite terms, Columbia Univ. Press, New York, 1948.

[ROS69] M. Rosenlicht, On the explicit solvability of certain transcendental equations, Inst. Hautes, Études Sci. Publ. Math. 36 (1969).

[ROS76] _ _ On Liouville's theory of elementary functions, Pacific J. Math. 65 (1976).

[SEI58] A. Seidenberg, Abstract differential algebra and the analytic case, Proc. Amer. Math. Soc. 9 (1958).

[SEI69] _ Abstract differential algebra and the analytic case. II, Proc. Amer. Math. Soc. 23 (1969).

[SIN76] M. F. Singer, Solutions of linear differential equations in function fields of one variable, Proc. Amer. Math. Soc. 54 (1976).

Department of Mathematics, Box 8205, North Carolina State University, Raleigh, North CAROLINA 27695

E-mail address: singer@math.ncsu.edu 